\title{
ALEXITHYMIA IN PATIENTS WITH HAEMOPHILIA. COMPARATIVE STUDY WITH THE HEALTHY POPULATION
}

\author{
Lavinia Hogea ${ }^{1}$, Laura Nussbaum ${ }^{2}$

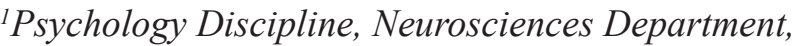 \\ University of Medicine and Pharmacy "V. Babes", Timisoara \\ ${ }^{2}$ Pedopsychiatry Discipline, Neurosciences Department, University of Medicine and Pharmacy "V. Babes", \\ Senior specialist Pediatric Psychiatry, Head of the Clinic of Child and Adolescent Psychiatry, Timisoara
}

\begin{abstract}
Introduction. Haemophilia is "a genetic transmitted disorder", it belongs to the category of rare diseases, illnesses that face devastating and incapacitating developments affecting each person beyond physical problems.

Material and method. 200 subjects were part of the study, they were divided into two equal groups (patients with hemophilia A and B $-50 \%$ and healthy population - 50\%), aged between 16 and 45 years. We used the medical history and measuring alexithymia with TAS-20 (Toronto Alexithymia Scale).

Results. The study conducted shows that there are statistically significant differences between hemophiliacs and non-hemophiliacs, hemophiliacs have higher levels of alexithymia compared to non-hemophiliacs.

Conclusions. The overall level of alexithymia was higher in patients with haemophilia than the other groups, including three subscales DIF (difficulty identifying emotions), DDF (difficulty in describing feelings) and EOT (externally oriented thinking).
\end{abstract}

Keywords: alexithymia, haemophilia

Note: The authors had an equal contribution in editing the present paper.

\section{INTRODUCTION}

The term alexithymia was first introduced in 1973. It comes from clinical observations of patients that show deficits in identifying and communicating emotional experiences, difficulties in distinguishing emotional states of emotional arousal of bodily sensations, and a poor life in imagination and fantasy.

Current conceptualizations describe alexithymia as a characteristic deficit in cognitive processing of emotional experience expressed through a limited ability to symbolize emotions and develop the emotional experience (1). Consequently, people with alexithymia may experience low awareness of the emotional and difficulty in regulating emotions and communication. Alexithymia has been studied extensively in adults as it is seen as a risk factor for a range of medical and health related problems. The presence and role of alexithymia were examined in various clinical disorders including post- traumatic stress disorder, panic disorder, eating disorders, somatoform disorders, alcoholism, substance abuse, asthma, anxiety and depression (2).

Alexithymia is generally regarded as consisting of the following dimensions: difficulty identifying and describing emotions, difficulties in distinguishing emotions of bodily sensations, imaginative life impoverished of fantasies and the trend of focusing on specific details of external events (3).

There are studies indicating that there are characteristics of alexithymia not only in classical psychosomatic disorders, but also in other severe and chronic somatic diseases $(4,5,6)$

\section{HYPOTHESIS}

Suppose there are significant differences in the level of alexithymia, meaning that subjects in clinical group will have a higher level of alexithymia compared with those in the control group. 


\section{MATERIAL AND METHODS}

The batch study includes both patients with hemophilia (No. 100) are in the evidence and Clinical treatment Center for evaluation and rehabilitation "Cristian Serban" of Buzias and Pediatric Clinic Timisoara, as well as healthy persons (No. 100), in different public institutions in Timisoara, and their selection was made in accordance with the criteria established for the whole study group.

For the conduct of the study, we used the medical history and measuring tool to alexithymia TAS20 (Toronto Alexithymia Scale).

\section{RESULTS}

To verify this hypothesis have used the results of the anamnesis, and for the measurement of the alexithymia have used the results of the TAS-20 (Toronto Alexithymia Scale).

The work assumption is one-sided. To test the hypothesis an ANOVA univariate 2 was made (people suffering from hemophilia, and people who do not suffer from hemophilia) X 3 (age-16-25 years, 26-35 years $36-45$ years old) to investigate the differences between groups and interaction effect. To validate this hypothesis we invoke the following quantitative arguments:

TABLE 1. Means and standard deviations on the alexithymia scale

\begin{tabular}{|l|c|c|c|c|c|}
\hline & $\begin{array}{c}\text { Hemo- } \\
\text { philiacs }\end{array}$ & $\begin{array}{c}\text { Non- } \\
\text { hemo- } \\
\text { philiacs }\end{array}$ & $\begin{array}{c}\text { Age } \\
16-25 \\
\text { age }\end{array}$ & $\begin{array}{c}\text { Age } \\
26-35 \\
\text { age }\end{array}$ & $\begin{array}{c}\text { Age } \\
36-45 \\
\text { age }\end{array}$ \\
\cline { 2 - 6 } & MAS & MAS & M AS & M AS & M AS \\
\hline Alexithymia & 69.83 & 55.76 & 62.19 & 61.48 & 67.71 \\
level & 12.43 & 9.73 & 13.05 & 13.21 & 13.06 \\
\hline
\end{tabular}

Table 1 shows averages and standard deviations for alexithymia to non-hemophiliacs and hemophiliacs respectively in age groups. At the alexithymia dimension, total scores ranged from 36 to $95(\mathrm{M}=62.79 ; \mathrm{AS}=13.18)$.

For testing the uniformity of dispersion I used the Levene test. The result is statistically significant, which indicates that the dispersions are heterogeneous, $\mathrm{F}(5,194)=2.601, \mathrm{p}<.05$.

TABLE 2. 3X2 factorial ANOVA summary table on the alexithymia scale

\begin{tabular}{|l|c|c|c|c|c|}
\hline $\begin{array}{l}\text { Source } \\
\text { dispersion }\end{array}$ & SS & Df & MS & $F$ & $P$ \\
\hline Group & 3839,481 & 1 & 3839,481 & $31,261^{* *}$ & $.000^{* *}$ \\
Age & 542,461 & 2 & 271,230 & 2,208 & .113 \\
Group X Age & 601,229 & 2 & 300,615 & 2,448 & .089 \\
Error & 23827,0 & 194 & 122,820 & & \\
Total & 34594,5 & 200 & & & \\
\hline
\end{tabular}

Nota: $* * p<.001$
From the three reports $\mathrm{F}$ presented in Table 2, only one is statistically significant: the main effect: F "lot" $(\mathrm{F}(1,194)=31.261, \mathrm{p}<.001,(\mathrm{M}=69.83$ versus $M=55.76)$ ). This means that the hypothesis is confirmed, so there are significant statistical differences between the hemophiliacs and nonhemophiliacs, hemophiliacs have a higher level of alexithymia compared to non-hemophiliacs, due to the fact that the main effect for batches (hemophiliacs versus non-hemophiliacs) is statistically significant.

The effect of the age variable on the alexithymia variable is statistically insignificant $F(2,194)=$ $2,20, p>.05$. This means that there were no significant differences found between the three age groups in hemophiliac patients $(\mathrm{M}=70.76$ on the group 16-25 years, $M=69.11$ on the group 26-35 years and $M=68.55$ on the group 36-45 years), compared to non-hemophiliacs $(\mathrm{M}=56.13$ on the group 16-25 years, $M=52.14$ on the group 26-35 years and $M=64.67$ on the group 36-45 years).

Also, the effect of the interaction group, age and alexithymia is insignificant statistically, F $(2,194)$ $=2.44, p>.05$, and neither the analysis of the interaction graph does not attest to the existence of an interaction of the variables contained in the study. (Fig. 1)

Summarizing, it appears that the overall level of alexithymia was higher in hemophiliac patients than in the other group, including three subscales DIF (difficulty in identifying emotions), DDF (difficulty in describing feelings) and EOT (externally oriented thinking).

We ask ourselves where these differences come from. To answer this question we must begin from the conceptualization of alexithymia as an attempt to block negative emotions associated with stress, then we could assume that the level of stress is higher in hemophiliac patients than in the control group subjects. This is plausible, because the subjects in the clinical trial are facing a chronic disabling disease with consequences on the quality of life.

Simultaneously, repeated bleedings can have negative consequences on the cognitive and emotional development. Of course, these interpretations are purely speculative. We can't really know what is the cause of the occurrence of these differences to the hemophiliac patients. Future studies could clarify the differences found.

However, in the scientific literature there are some studies that come to reinforce earlier observations. 


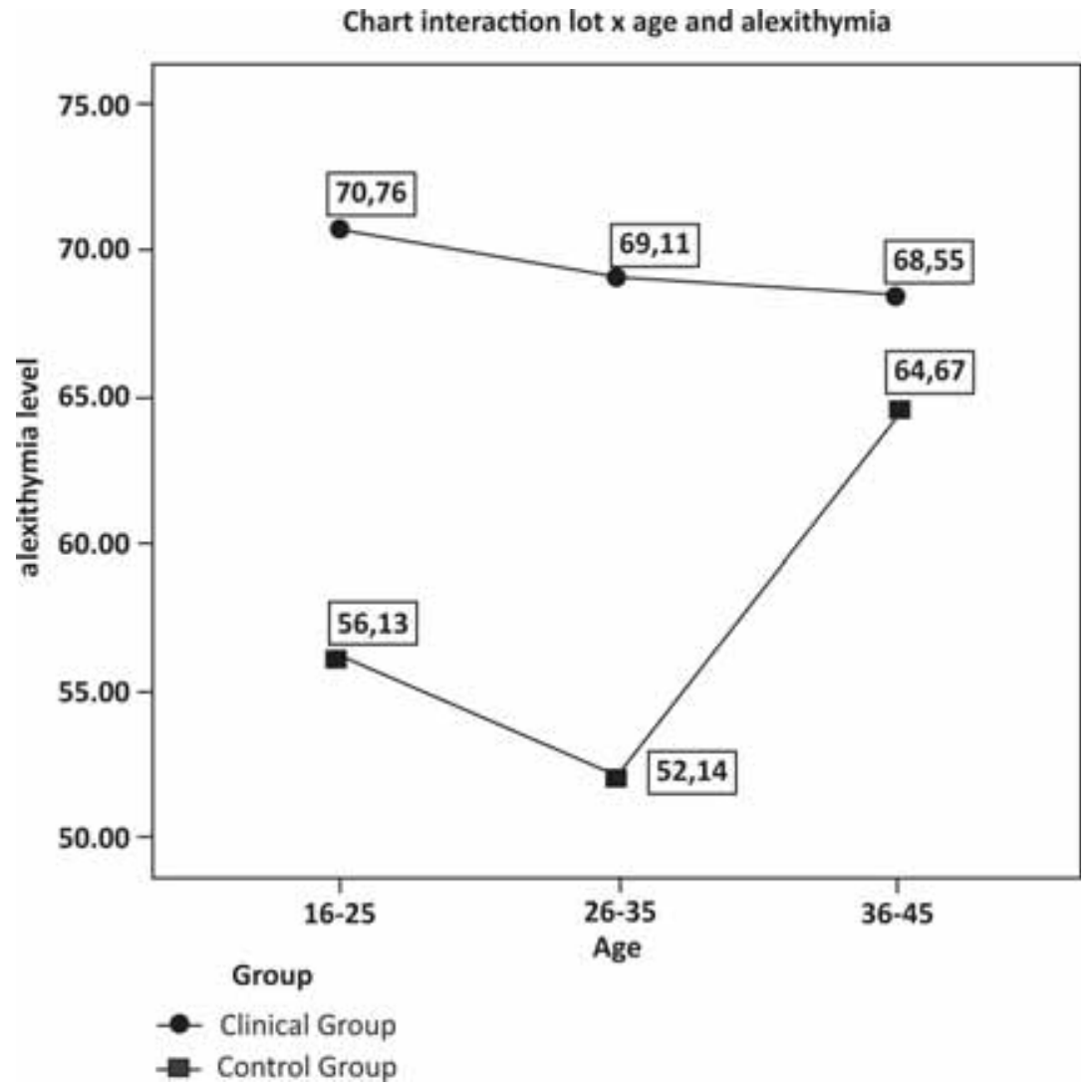

FIGURE 1. Graphic interaction group $X$ age and alexithymia

In a study from Cincinati performed on children with hemophilia, social functions, emotional and behavioral problems were investigated, so it has been reported that the hemophiliac patients exhibit emotional well-being difficulties, reduced social relationships, as well as depressive symptomatology (7).

Chen has conducted a study investigating alexithymia, introversion and extraversion on 1,788 subjects both men and women with an average age of 20.44 years. The results reveal a link between alexithymia and introversion. In other words, introvert individuals present an increased alexithymia score and face increased stress related to social life in comparison with the general population.

This study comes in support of the idea that hemophiliac patients living a life of isolation and with an increased level of stress may exhibit a higher score of alexithymia, although introversion was not measured here.

Also, the higher or lower stress level, correlated with a higher or lower perceived social support may lead to differences in the processing of emotional experience and in the emotions regulation, so to differences in terms of experiencing alexithymia.

\section{CONCLUSIONS}

Regarding the proposed hypothesis for investigating the difference, on the alexithymia dimen- sion, between the two groups, it has been confirmed: $\mathrm{F}(1.194)=31.261, \mathrm{p}<.001,(\mathrm{M}=69.83$ versus $M=55.76)$. It seems that the alexithymia level was higher in hemophiliac patients than in the other group, the differences being statistically relevant in relation to the overall score of alexithymia. Studies from the scientific literature support the idea that repeated bleedings may have negative consequences over the cognitive and emotional development. Moreover, the life of isolation and the increased level of stress may exhibit a higher score of alexithymia. The differences obtained between the two studied groups, may have relevance especially from the point of view of practitioners in the field, who can guide their interventions depending on the noticed differences.

As can be seen, hemophilia affects every person beyond physical problems. In order to optimize the efforts to facilitate the patients' health, it is important that alongside medical treatment they should also be given psycho-social care, as an integral part of a multidisciplinary approach.

People with coagulation disorders, live everywhere around the value of world. Professionals from hemophilia centers play an important role in critical moments in the life of patients, giving them the essential tools to help them improve their lives, to participate actively in the treatment and to be able to manage the disease. 


\section{REFERENCES}

1. Kooiman C.G., The status of alexithymia as a risk factor in medically unexplained physical symptoms. Compr Psychiatry, 1998;

2. Aino K.M., et al., Alexithymia and Health-Related Quality of Life in a General Population, 2009);

3. Sifneos P.E., Alexithymia: past and present, 1996;

4. Taylor G.J., et al., Alexithymia and somatic complaints in psychiatric outpatients, 1992;
5. Parker J.D., et al., Alexithymia in panic disorder and simple phobia: a comparative study, 1993;

6. Bankier B., et al., Alexithymia in DSM-IV disorder: comparative evaluation of somatoform disorder, panic disorder, obsessivecompulsive disorder, and depression 2001;

7. Trzepacz A.M., Social, emotional, and behavioral functioning of children with hemophilia, 2003, 225-32. 